\title{
ON THE LAPLACE TRANSFORM OF A TEMPERATE DISTRIBUTION SUPPORTED BY A CONE
}

\author{
BENT E. PETERSEN
}

\begin{abstract}
The temperate distributions supported by a closed convex salient cone are characterized by explicit polynomial growth of the Laplace transform at infinity and at the boundary of the cylinder over the dual cone. This result is then used to characterize by their Laplace transforms the smooth kernels of degree $\lambda-n$ where $\lambda$ is positive.
\end{abstract}

Let $\Gamma$ be a closed convex cone in $R^{n}$ with vertex at the origin. The dual cone $\Gamma^{+}$is defined by

$$
\Gamma^{+}=\left\{\xi \in R^{n} \mid\langle\xi, x\rangle \geqq 0, x \in \Gamma\right\} .
$$

Clearly $\Gamma^{++}=\Gamma$. The interior $\Gamma_{0}^{+}$of $\Gamma^{+}$is nonempty if and only if $\Gamma$ is salient, i.e. contains no one dimensional subspace. If $\xi \in \Gamma_{0}^{+}$then $\delta=$ $\delta(\xi)=\inf \{\langle\xi, x\rangle|x \in \Gamma| x \mid,=1\}$ is equal to the distance from $\xi$ to the boundary of $\Gamma^{+}$and $\Gamma \subseteq\left\{x \in R^{n}|\langle\xi, x\rangle \geqq \delta| x \mid\right\}$.

Suppose now that $\Gamma$ is closed convex and salient. Let $T$ be a temperate distribution with support contained in $\Gamma$ and let $F$ be the Laplace transform of $T$. Then Streater and Wightman [4] attribute to unpublished remarks of $L$. Gårding the following results.

(i) For each $\xi_{0} \in \Gamma_{0}^{+}$there exists a polynomial $P$ such that

$$
\left|F\left(\xi_{0}+\xi+i \eta\right)\right| \leqq|P(\xi+i \eta)|, \quad \xi \in \Gamma^{+}, \eta \in R^{n} .
$$

(ii) For each compact subset $K$ of $\Gamma_{0}^{+}$there is a polynomial $P_{K}$ and an integer $r_{K}$ such that

$$
|F(t \xi+i \eta)| \leqq\left|P_{K}(\eta)\right| t^{-r_{K}}, \quad \xi \in K, \eta \in R^{n}, 0<t<1 .
$$

Conversely, each holomorphic function in $\Gamma_{0}^{+}+i R^{n}$ which satisfies (i) and (ii) is the Laplace transform of a temperate distribution with support in $\Gamma$. Proofs may be found in [4] for the case of a special cone $\Gamma$ in $R^{4 n}$ with the property that $\Gamma^{+}=\Gamma$. In our first theorem we prove these results in

Received by the editors December 2, 1971.

AMS 1970 subject classifications. Primary 44A10, 46F10; Secondarv 32A07, 35A22. Key nords and phrases. Laplace transform, temperate distribution.

c. American Mathematical Society 1972 
such a way that we may see precisely how $P$ depends on $\xi_{0}$ and how $P_{K}$ and $r_{K}$ depend on $K$.

If $\phi \in \mathscr{S}\left(R^{n}\right)$ and $m, N \geqq 0$ where $N$ is an integer we define

$$
\|\phi\|_{m, N}=\max _{|\alpha| \leqq N} \sup _{x}\left|(1+|x|)^{m} D^{\alpha} \phi(x)\right| .
$$

Then these norms determine the topology of $\mathscr{S}\left(R^{n}\right)$. Our sign and other conventions regarding the Laplace transform follow those of Schwartz [2]. In the following theorem $m$ need not be an integer.

TheOrem 1. Let $\Gamma$ be a closed convex salient cone in $R^{n}$. If $T$ is a temperate distribution continuous relative to the norm $\|\cdot\|_{m . N}$ whose support is contained in $\Gamma$, then the Laplace transform $F$ of $T$ is a holomorphic function in the cylinder $\Gamma_{0}^{+}+i R^{n}$ and there exists a constant $C>0$ such that

$$
|F(\xi+i \eta)| \leqq C\left(1+\delta(\xi)^{-m-N}\right)(1+|\xi+i \eta|)^{N}
$$

for each $\xi \in \Gamma_{0}^{+}$and $\eta \in R^{n}$.

Conversely if $F$ is a holomorphic function in $\Gamma_{0}^{+}+i R^{n}$ which satisfies (1) then $F$ is the Laplace transform of a temperate distribution with support in $\Gamma$.

We observe if we apply (1) to $t^{\lambda} F(t \xi+i t \eta)$ where $t=\delta(\xi)^{-1}$ we obtain the following result.

COROLlaRY. If $T$ is homogeneous of degree $\lambda-n$ then

$$
|F(\xi+i \eta)| \leqq C \delta(\xi)^{-\lambda}\left(1+\delta(\xi)^{-1}|\xi+i \eta|\right)^{N}
$$

for each $\xi \in \Gamma_{\mathbf{0}}^{+}$and $\eta \in R^{n}$.

Our second theorem characterizes $C^{\infty}$ kernels by their Laplace transforms. This theorem was motivated by the possibility that it may prove to be relevant to an interesting conjecture of K. T. Smith [3]. By a $C^{\infty}$ kernel of degree $\lambda-n$ where $\lambda$ is a real number we mean a $C^{\infty}$ function $K$ on $R^{n}-(0)$ homogeneous of degree $\lambda-n$. In general the Hadamard finite part Pf $K$ defines a temperate distribution and $\operatorname{Pf} K$ is homogeneous of degree $\lambda-n$ if $\lambda \neq 0,-1,-2, \cdots$. In case $\lambda>0$ then $K$ is locally integrable and defines a temperate distribution continuous relative to the norm $\|\cdot\|_{\lambda+1,0}$.

THEOREM 2. Let $\Gamma$ be a closed convex salient cone in $R^{n}$. Let $K$ be a $C^{\infty}$ kernel of degree $\lambda-n$ where $\lambda>0$. Suppose the support of $K$ is contained in $\Gamma$ and let $F$ be the Laplace transform of $K$. Then for each integer $m \geqq 0$ there exists a constant $C_{m}>0$ such that if $|\beta|=m$ then

$$
\left|D^{\beta} F(\xi+i \eta)\right| \leqq C_{m} \delta(\xi)^{-\lambda}|\xi+i \eta|^{-m}
$$


for each $\xi \in \Gamma_{0}^{+}$and $\eta \in R^{n}$. Conversely if $F$ is a holomorphic function in $\Gamma_{0}^{+}+i R^{n}$ homogeneous of degree $-\lambda<0$ such that (2) holds then $F$ is the Laplace transform of a $C^{\infty}$ kernel of degree $\lambda-n$ with support contained in $\Gamma$.

We begin by giving the proof of Theorem 2. If $\lambda-|\alpha|+|\beta|>0$ then $D^{\alpha} x^{\beta} K$ is a $C^{\infty}$ kernel of degree $\lambda-|\alpha|+|\beta|-n>-n$. Hence by the Corollary its Laplace transform is bounded by $C \delta(\xi)^{-\lambda+|\alpha|-|\beta|}$. If we denote temporarily by $\tilde{D}$ differentiation in the distribution sense then $D^{\alpha} x^{\beta} K-$ $\tilde{D}^{\alpha} x^{\beta} K$ is supported by the origin and is homogeneous of degree strictly larger than $-n$ and therefore is zero. Thus the differentiation in $D^{\alpha} x^{\beta} K$ is in the distribution sense and hence the Laplace transform of $D^{\alpha} x^{\beta} K$ is $(\xi+i \eta)^{\alpha}(-D)^{\beta} F$. It follows that if $\lambda-|\alpha|+|\beta|>0$ then

$$
\left|(\xi+i \eta)^{\alpha} D^{\beta} F(\xi+i \eta)\right| \leqq C \delta(\xi)^{-\lambda+|\alpha|-|\beta|} .
$$

Now let $m \geqq 0$ be an integer. Then

Thus if $|\beta|>m-\lambda$ then

$$
|\xi+i \eta|^{m} \leqq\left(\sum\left|\xi_{j}+i \eta_{j}\right|\right)^{m}=\sum_{|\alpha|=m} \frac{m !}{\alpha !}\left|(\xi+i \eta)^{\alpha}\right| .
$$

$$
\left|D^{\beta} F(\xi+i \eta)\right| \leqq C_{\beta} \delta(\xi)^{-\lambda+m-|\beta|}|\xi+i \eta|^{-m}
$$

and (2) certainly follows.

Conversely suppose $F$ is holomorphic in $\Gamma_{0}^{+}+i R^{n}$, is homogeneous of degree $-\lambda<0$, and satisfies (2). By Theorem $1, F$ is the Laplace transform of a temperate distribution $K$ with support in $\Gamma$. An easy computation shows that $K$ is homogeneous of degree $\lambda-n$.

Let $\xi \in \Gamma_{0}^{+}$. According to (2) if $|\beta| \geqq|\alpha|+n+1$ then

$$
\left|(\xi+i \eta)^{\alpha} D^{\beta} F(\xi+i \eta)\right| \leqq C(1+|\eta|)^{-\cdots 1}
$$

where $C$ depends on $\alpha, \beta$ and $\xi$. Thus $(\xi+i \eta)^{\alpha} D^{\beta} F(\xi+i \eta)$ is integrable as a function of $\eta$ and hence its inverse Fourier transform is continuous, i.e. $e^{-\langle\xi \cdot\rangle} D^{\alpha}\left((-x)^{\beta} K\right)$ is continuous. It follows that if $|\beta| \geqq k+n+1$ then $x^{\beta} K$ is of class $C^{k}$. Hence $K$ is of class $C^{\infty}$ on $R^{n}-(0)$.

We now proceed to the proof of Theorem 1 .

If $\xi \in \Gamma^{+}$then $e^{-\langle\xi, x\rangle}$ and all its derivatives are bounded on some open half space which contains $\Gamma$ and hence $e^{-\langle\xi \cdot \cdot\rangle} T$ is temperate. Its Fourier transform is a function if $\xi \in \Gamma_{0}^{+}$, and if $F(\xi+i \eta)=\left(e^{-\langle\xi, \cdot\rangle} T\right)^{\wedge}(\eta)$ then $F$ is the Laplace transform of $T$ and is holomorphic in $\Gamma_{0}^{+}+i R^{n}$. See Schwartz [2, Chapter 8].

Choose $\theta \in \mathscr{D}\left(R^{n}\right)$ such that $\theta(x)=1$ for $|x| \leqq 1$. Then $\theta T \in \mathscr{E}^{\prime \prime}\left(R^{n}\right)$. The Laplace transform of $\theta T$ is an entire function $F_{1}$ and $F_{1}(\xi+i \eta)=$ $(\theta T)^{\wedge}(\eta-i \xi)$. Hence by the Paley-Wiener theorem [1, p. 211],

$$
\left|F_{1}(\xi+i \eta)\right| \leqq C(1+|\xi+i \eta|)^{N} e^{H(\xi)}
$$


since $\theta T$ has order $\leqq N$. Here $H$ is the support function of supp $\theta T$. In particular if $\xi \in \Gamma^{+}$then

$$
H(-\xi)=\sup \{\langle-\xi, x\rangle \mid x \in \operatorname{supp} \theta T\} \leqq 0
$$

and so

$$
\left|F_{1}(\xi+i \eta)\right| \leqq C(1+|\xi+i \eta|)^{N}, \quad \xi \in \Gamma^{+}, \eta \in R^{n} .
$$

Now consider $(1-\theta) T$ and denote its Laplace transform by $F_{2}$ so $F=F_{1}+F_{2}$. Let $h \in \mathscr{E}(R)$ be such that

$$
\begin{aligned}
h(t)=0 & \text { if } t \leqq 1, \\
=1 & \text { if } t \geqq \frac{6}{5} .
\end{aligned}
$$

Let $\xi \in \Gamma_{0}^{+}, \delta=\delta(\xi)$, and define

$$
\psi(x)=h\left(4 \delta^{-1}\langle\xi, x\rangle\left(1+|x|^{2}\right)^{-1 / 2}\right) .
$$

Then $\psi \in \mathscr{E}\left(R^{n}\right)$ and we have: if $\psi(x) \neq 0$ then $\left.\langle\xi, x\rangle\right\rangle(\delta / 4)|x|$. If $U=$ $\left\{x \in R^{n}|| x \mid>\frac{3}{4}\right.$ and $\left.\langle\xi, x\rangle>(\delta / 2)|x|\right\}$ then $U$ is an open neighborhood of $\Gamma \cap \operatorname{supp}(1-\theta)$. If $x \in U$ then

$$
4 \delta^{-1}\langle\xi, x\rangle\left(1+|x|^{2}\right)^{-1 / 2} \geqq 2|x|\left(1+|x|^{2}\right)^{-1 / 2} \geqq \frac{6}{5}
$$

since $|x|>\frac{3}{4}$, and hence $\psi(x)=1$. Thus $\psi$ is 1 on an open neighborhood of $\Gamma \cap \operatorname{supp}(1-\theta)$.

Now we claim that $\phi=e^{\langle\langle\xi+i \eta \cdot \cdot\rangle} \psi$ belongs to $\mathscr{S}\left(R^{n}\right)$. Indeed if $M \geqq 0$ is an integer and $k \geqq 0$, by the Leibnitz formula, if $|\alpha| \leqq M$ then

$$
\left|D^{\alpha} \phi(x)\right| \leqq C \sum\left|D^{\beta} e^{-\langle\xi+i \eta \cdot x\rangle}\right| \cdot\left|D^{\gamma} \psi(x)\right|
$$

where the sum is taken over $|\beta|+|\gamma| \leqq M$. On the support of $\psi$ we have $\langle\xi, x\rangle \geqq(\delta / 4)|x|$ and hence

$$
\left|D^{\beta} e^{-\langle\xi+i \eta, x\rangle}\right| \leqq C(1+|\xi+i \eta|)^{|\beta|} e^{-(\delta / 4)|x|}
$$

on the support of $\psi$. Clearly we may choose a common bound for the first $M$ derivatives of $h$ on the real line and therefore if $|\gamma| \leqq M$ then

$$
\left|D^{\gamma} \psi(x)\right| \leqq C\left(1+\delta^{-1}|\xi|\right)^{|\gamma|} \leqq C\left(1+\delta^{-|\gamma|}\right)(1+|\xi|)^{|\gamma|}
$$

where $C$ does not depend on $\delta$ or $\xi$. Thus

Now clearly

$$
\|\phi\|_{k, M} \leqq C(1+|\xi+i \eta|)^{M} \max _{l \leqq M}\left(1+\delta^{-l}\right) \sup _{x}(1+|x|)^{k} e^{-(\delta / 4)|x|} .
$$

$$
e^{-(\delta ; 4)|x|}(1+|x|)^{k} \leqq 1+\left(4 k \delta^{-1}\right)^{k}
$$


and

$$
\max _{l \leqq M}\left(1+\delta^{-k}\right)\left(1+\delta^{-l}\right) \leqq 4\left(1+\delta^{-k-\lambda l}\right) .
$$

Thus

$$
\|\phi\|_{k, \lambda} \leqq C\left(1+\delta(\xi)^{-k-M}\right)(1+|\xi+i \eta|)^{M}
$$

where $C$ depends only on $k$ and $M$.

Now let $\theta_{p}(x)=\theta\left(p^{-1} x\right), p=1,2, \cdots$. Then $\theta_{p} T$ is a bounded sequence in $\mathscr{S}^{\prime}\left(R^{n}\right)$ and $\theta_{p} T \rightarrow T$ in $\mathscr{D}^{\prime}\left(R^{n}\right)$. Hence by the Banach-Steinhaus theorem $\theta_{p} T \rightarrow T$ in $\mathscr{S}^{\prime}\left(R^{n}\right)$. Let $G_{p}$ be the Laplace transform of $(1-\theta) \theta_{p} T$. Then $G_{p}$ is an entire function and

$$
G_{p}(\xi+i \eta)=\theta_{p} T\left((1-\theta) e^{-\langle\xi+i \eta \cdot \cdot\rangle}\right) .
$$

Now if $\xi \in \Gamma_{0}^{+}$then $\psi$ is 1 in a neighborhood of $\operatorname{supp}(1-\theta) T$ and hence

$$
G_{p}(\xi+i \eta)=\theta_{p} T((1-\theta) \phi) .
$$

Since $(1-\theta) \phi \in \mathscr{I}^{\prime}\left(R^{n}\right)$ we have

$$
\lim _{n \rightarrow \infty} G_{p}(\xi+i \eta)=T((1-\theta) \phi) .
$$

Now $\left|D^{\alpha} \theta_{p}(x)\right|=p^{-|\alpha|}\left|\left(D^{\alpha} \theta\right)\left(p^{-1} x\right)\right| \leqq \sup _{x}\left|D^{\alpha} \theta(x)\right|$ and therefore

$$
\left|G_{p}(\xi+i \eta)\right| \leqq C\|\phi\|_{m, N} \leqq C\left(1+\delta^{-m-N}\right)(1+|\xi+i \eta|)^{N}
$$

where $C$ does not depend on $p$. Then by the Lebesgue dominated convergence theorem the convergence in (6) is in the sense of $\mathscr{S}_{\eta}^{\prime}\left(R^{n}\right)$. But $G_{\nu}(\xi+i \eta)=\left(\theta_{p} e^{-\langle\xi \cdot \cdot\rangle}(1-\theta) T\right)^{\wedge}$ converges in $\mathscr{S}_{\eta}^{\prime}\left(R^{n}\right)$ to $\left(e^{-\langle\xi \cdot\rangle}(1-\theta) T\right)^{\wedge}=$ $F_{2}(\xi+i \eta)$ and therefore we have $F_{2}(\xi+i \eta)=T((1-\theta) \phi)$. It follows that

$$
\left|F_{2}(\xi+i \eta)\right| \leqq C\|\phi\|_{m, N} .
$$

Statements (4), (5) and (7) yield (1).

The converse follows immediately from the remarks preceding Theorem 1. Indeed our hypotheses have the appearance of being stronger than (i) and (ii) since $\delta\left(\xi_{0}+\xi\right) \geqq \delta\left(\xi_{0}\right)$. In outline the proof goes as follows. That $F$ is the Laplace transform of a distribution $T$ is immediate [2, p. 306]. That the support of $T$ is contained in $\Gamma^{++}=\Gamma$ follows by a theorem of $\mathrm{J}$. L. Lions [2, p. 310]. Finally that $T$ is temperate is shown by the argument of L. Gårding [4, p. 62]. 


\section{REFERENCES}

1. W. F. Donoghue, Jr., Distributions and Fourier transforms, Academic Press, New York, 1969.

2. Laurent Schwartz, Théorie des distributions, Publ. Inst. Math. Univ. Strasbourg, no. 9-10, Nouvelle édition, entièrement corrigee, refondue et augmentée, Hermann, Paris, 1966. MR 35 \#730.

3. K. T. Smith, Formulas to represent functions by their derivatives, Math. Ann. 188 (1970), 53-77.

4. R. F. Streater and A. S. Wightman, PCT, spin and statistics, and all that, Benjamin, New York, 1964. MR 28 \#4807.

Department of Mathematics, Oregon State University, Corvallis, Oregon 97331 